\title{
The Ayurvedic Perspective on the Etiopathogenesis of Neurodegeneration
}

\section{Jibin Joy*}

Translational Scientific Research, Mundomthanath House, Poothrikka P.O, Puthencruz via Ernakulam, Kerala-682308, India

*Corresponding author: Jibin Joy, Translational Scientific Research, Mundomthanath House, Poothrikka P.O, Puthencruz via Ernakulam, Kerala-682308, India, Tel: +91-0484-2764941; E-mail: jibinjoyindia@gmail.com

Rec date: Sep 06, 2015; Acc date: Sep 26, 2015; Pub date: Sep 28, 2015

Copyright: $\odot 2015$ Jibin Joy. This is an open-access article distributed under the terms of the Creative Commons Attribution License, which permits unrestricted use, distribution, and reproduction in any medium, provided the original author and source are credited.

\begin{abstract}
The homeostatic signaling system in Homo sapiens is highly complex involving diverse physiological processes which are what enables the biological regulation of life. Human body is a highly dynamic system, the normal functioning of which is coordinated and maintained by a well-established mechanism of homeostatic regulation. Disorder genesis and progression has an underlying disruption in homeostatic regulation, even though pathophysiology and clinical presentations may vary. Progressive neurodegenerative conditions are an outcome of disrupted homeostatic regulation, which triggers accumulation of dysfunctions and impairment of neuroplasticity.

Ayurveda considers neurodegenerative conditions to be brought by the imbalance of 'Vata', the energy that moves through the brain and the nerves. The scholars behind classical ayurvedic literature considered neural impulses to be a kind of air traveling through the body, controlling both voluntary and involuntary functions. Oxygen is the real time mediator of homeostatic signaling system and defective oxygen transport; thereby impairment in oxygenation is the prime underlying factor in neurodegenerative conditions. The broad spectrum therapeutic effects demonstrated by Withania Somnifera or Ashwagandha are a result of its profound mechanism of enhancement of oxygenation. Also the ayurvedic branch of medicine known as 'Rasa Shastra' uses 'Bhasma', traditionally prepared Nano medicines, some of which shows influential enhancement in oxygenation.
\end{abstract}

Keywords: Neurodegeneration; Synaptogenesis; Alzheimer's disease; Homeostatic regulation; Parkinson's disease

\section{Abbreviations \\ COX: Cytochrome $C$ Oxidase; Oxphos-Oxidative phosphorylation; NFT: Neuro fibrillary Tangles; AD: Alzheimers Disease; PD: Parkinsons Disease; DLB: Dementia Lewy Body; CNS: Central Nervous System; Gpx: Erythrocyte glutathione peroxidase; SOD: Superoxide dismutase; CAT: Catalase; MB: Methylene Blue}

\section{Introduction}

The classical ayurvedic literatures on 'Vata Raktha Chikitsa' [1] clearly suggests that complex disorders affecting nervous system originates from an underlying imbalance of 'vata'. Furthermore, Ayurveda considers human body as a vehicle for spiritual experience which is suggestive of the fact that human consciousness is not locally stored in nervous system. This co-relates with the studies on the underlying mechanisms of cytoskeletal signaling [2]. It has been favored by quantum theory perspectives, that fundamental disturbances in consciousness arises from an underlying disturbance in cytoskeletal system. The micro tubule based concepts of consciousness has been validated in several clinical research studies and implicate aberrant micro tubules as a pathogenetic mechanism in disorders affecting consciousness [3-5]. The cytoskeletal system exhibits dynamic behavior, and it does interacts with hundreds of associated proteins, molecular motors, cross linkers. Also involved in many cell signalling pathways and cytokinesis [6-8]. As described in classical ayurvedic literature, when it comes to the five elements of creation, air is considered to be related to movement and electrical energy. And hence it is suggestive of the fact that oxygen is the fundamental electron transport mechanism in the universe. It's the way through which the universe is monitoring, recording, maintaining internal stability, obtaining coordinated responses and is managing the ecology of life. All processes of integration and coordination of function in the ecology of life, whether mediated by nervous and hormonal systems comes under homeostatic regulation. The primary target and aim of every disease and disorder in the ecology of life is to impair oxygenation the outcome of which is the disruption of homeostatic regulation. An illness or disease thrives only in an oxygen deficient environment. Impairment in oxygenation implies disruption of homeostatic regulation [9]. Furthermore therapeutic approaches used in ayurvedic medicinal systems for longevity, vitality are suggestive of the underlying mechanism of enhancement of oxygenation. Withania Somnifera or 'Ashwagandha' is showing a similar therapeutic potential. Also certain nanomedicines used in ayurvedic medicinal system prepared in traditional manner which are referred to as 'Bhasma' are demonstrating similar potential [10].

Since oxygen is the mediator of electron-transport mechanism, a dysfunction in the electron-transport mechanism is suggestive of disrupted homeostatic regulation. And a lot of experimental and clinical studies favor a major disruption in homeostatic regulation in neurodegenerative conditions. Respiratory chain deficits, mitochondria dysfunction, exacerbated reactive oxygen species production, low hemoglobin levels, deficiency in the terminal complex of the mitochondrial electron transport chain-cytochrome $c$ oxidase (COX), defects in oxidative phosphorylation (Oxphos) has been observed in the pathophysiology of neurodegenerative conditions. These observations suggests that impairment in oxygenation is at the core of neurodegeneration. Its quite interesting that a rejected theory on neurodegeneration, which had considered 'circle of williesatherosclerosis' as a contributing factor to Alzheimer's disease, neuritic 
plaques and neurofibrillary tangles. This was conceptualized based on vascular hypothesis. From this also it can be presumed that impairment in oxygenation can contribute to pathophysiology of neurodegeneration. The mechanisms of oxygen sensing and homeostatic regulation is contributed by several intracellular signaling systems. Even a slight disruption in the normal environment can result in mild to severe disruption on homeostatic regulation.

\section{Background}

Homeostasis refers to stability, balance or equilibrium; its an attempt to maintain a constant internal environment. Maintaining a stable internal environment requires constant monitoring and adjustments as conditions change. This adjusting of physiological systems within the body is called homeostatic regulation. Since oxygen is the mediator of homeostatic regulation, defective oxygen transport causes disruptions in homeostatic regulation. There are several underlying physiological processes that eventually contributes in maintaining homeostatic regulation which governs life. Imbalances in these underlying processes as a result of impaired oxygenation there by results in disrupted homeostatic regulation.

When we look into the pathogenesis of neurodegeneration, it can be seen that a wide array of neurodegenerative conditions are caused by intraneuronal accumulations of intracellular or extracellular protein aggregates. Disorder genesis and pathological changes involve proteins which are subject of altered post-translational modifications, including truncation and cleavage, which promote abnormal solubility, aggregation, and fibril formation. As a result, most degenerative diseases of the nervous system including Alzheimer's disease (AD) and Parkinson's disease (PD) are recognized by the end-products accumulated in the brain. These abnormal aggregates form amyloid plaques and neurofibrillary tangles (NFT) composed of hyperphosphorylated tau in $\mathrm{AD}$, pre-tangles and alpha synuclein inclusions named Lewy bodies in PD and related synucleinopathies such as dementia with Lewy bodies (DLB) [11-16]. This inturn occurs as a result of disruption in homeostatic regulation governing life. While in the case of Amyotrophic Lateral Sclerosis whose clinical features suggests a pathogenesis of upper and lower motor neuron degeneration. The motor neurons serve as the controlling units and vital communication links between the nervous system and the voluntary muscles of the body. And hence their degeneration can result in wide range of disabilities. Even though the disease doesn't affect a person's mind or intelligence, several recent studies suggests that ALS can also cause decline in cognitive functioning. When it comes to homeostatic regulation, it encompasses all forms of integration and coordination of functions, whether mediated by nervous and hormonal systems. And so impairment in homeostatic regulation, suggests a vast array of underlying factors that contributes in the pathophysiology and progression, such as impairment in the regulation through HPA and HPG axis. Several studies reported the dysfunction of prominent functions performed by neuroendocrine systems in the pathophysiology of neurodegeneration [17-21].

Homeostatic regulation of neuronal excitability refers to the collective phenomena by which neurons alter their intrinsic or synaptic properties to maintain a target level of electrical activity. Dysfunction of these fundamental processes could thereby contribute to the pathophysiology of neuropsychiatric presentations as observed in neurodegenerative conditions. [22,23]. The term neuroplasticity relates to diverse processes of vital importance by which the brain perceives, adapts and responds to a variety of internal and external stimuli. The manifestations of neuroplasticity in the adult CNS have been characterized as including alterations of dendritic function, synaptic remodeling, long-term potentiation (LTP), axonal sprouting, neurite extension, synaptogenesis, and even neurogenesis [24,25]. There are several underlying mechanisms which regulates neuroplasticity which is a part of the homeostatic regulation system. Disruptions in these mechanisms of homeostatic regulation contributed by impairment in oxygenation thereby results in dysfunctions in neuroplasticity. Previous studies shows the presence of hemoglobin alpha-chain and beta-chain in neurons of the rodent and human brain thus indicating that hemoglobin is a normal component of nerve cells and that hemoglobin may play a role in intraneuronal oxygen homeostasis.[11]. Also several studies have suggested deficiency in the terminal complex of the mitochondrial electron transport chain, cytochrome $c$ oxidase (COX), in platelet mitochondria of Alzheimer's disease (AD) patients [26]. Also low hemoglobin level has shown to have been associated with AD [27]. Parkinson's disease also has been associated with defects in oxidative phosphorylation (Oxphos) [28]. Also a study reported defects of respiratory chain complexes were considered as possible pathogenetic mechanisms in Parkinson's disease (PD) [29,30]. Multiple studies also reported an association between anemia experienced early in life and the later development of Parkinson disease [31].

Mitochondria are one of the major producers of ROS and are also the main targets of oxidative damage, mitochondrial dysfunction and exacerbated ROS production is generally associated with neurodegenerative disorders. It has been observed that mitochondrial dysfunction, inflammation, iron dysregulation and apoptosis have a crucial role in the pathogenesis of several neurodegenerative disorders. [32-34]. Iron, Copper, Zinc and Magnesium has prominent roles in biological electron transport and oxygen transportation. Iron $(\mathrm{Fe})$ is an essential element that is imperative for the redox-driven processes of oxygen transport, electron transport, and DNA synthesis. Previous studies which evaluated the status of plasma essential trace elements magnesium $(\mathrm{Mg})$, copper $(\mathrm{Cu})$, zinc $(\mathrm{Zn})$, iron $(\mathrm{Fe})$ and selenium $(\mathrm{Se})$ concentrations and their some related antioxidant enzyme activities, erythrocyte glutathione peroxidase $(\mathrm{GPx})$, superoxide dismutase (SOD), and catalase (CAT) activities in patients with Alzheimer's disease $(\mathrm{AD})$ suggested alterations in essential trace elements and their related enzymes may play a role in the etiopathogenesis of AD. Also, there is a defect in the antioxidant defense system, which may lead to oxidative damage in patients with AD [35]. However it has been observed that, in the absence of appropriate storage or chelation, excess-free $\mathrm{Fe}$ readily participates in the formation of toxic-free radicals, inducing oxidative stress and apoptosis. The brain shares with other organs the need for a constant and readily available supply of iron and has a similar array of proteins available to it for iron transport, storage, and regulation. In a number of common neurodegenerative disorders, there appears to be an excess accumulation of iron in the brain that suggests a loss of the homeostatic mechanisms responsible for regulating iron in the brain. The disruptions in such mechanisms of homeostatic regulation is brought by the impairment in oxygenation. As a result of a loss in iron homeostasis, the brain becomes vulnerable to iron-induced oxidative stress. The disrupted expression or function of proteins involved in iron metabolism increases the concentration of iron in the brain. Disturbances can happen at any of several stages in iron metabolism. Increased brain iron triggers a cascade of deleterious events that lead to neurodegeneration [32-34]. Also this disrupted expression or 
functions of proteins thereby suggests a disruption in homeostatic regulation governing life.

Also it's an interesting fact to know that several medications that have proven to improve oxygenation provided benefits in neurodegenerative conditions including Alzheimer's Disease. This is suggestive of benefits obtained from 'Methylene Blue' (MB). Several studies suggested MB hold a therapeutic potential for Alzheimer's disease. MB activated Amyloid Beta clearance, increases in proteasome function and ameliorated AD-like pathology in-vivo. [36,37]. In Ayurvedic medicinal system, therapeutic approaches used in neurodegenerative conditions suggest a similar mechanism of action, which is by enhancing oxygenation. One of the best candidate showing such a potential is 'Withania Somnifera' or 'Ashwagandha' which have shown to have potent hematonergic, immune enhancing effects [38-43]. Withania Somnifera is known to alter the oxidative stress markers of the body, found to have neuroprotective activity, free radical scavenging activity and has found to significantly reduce the lipid peroxidation and increase the superoxide dismutase (SOD) and catalase activities. Ashwagandha for its benefits have been used since thousands of years in Ayurvedic medicinal systems for neurodegenerative conditions. The synergistic effects thus produced by Ashwagandha is suggesting its profound mechanism of enhancement of oxygenation. Also it has been observed that drinking oxygenated water has shown to enhance the immune status [44]. This is suggestive of the fact that enhancing oxygenation is improving homeostatic regulation. The involvement of glymphatic system in neurodegenerative conditions are very prominent. Enhancement of oxygenation can activate glymphatic clearance pathways and can produce multiple target actions which has promising effects in delaying progression and furthermore in the promotion of neurogenesis. Through enhancement of oxygenation, the disruptions in homeostatic mechanisms governing life can be re-established which can induce healing and can be used in prevention of diseases. This is also suggestive of the benefits associated with a vegetarian diet. A diet rich in green leafy vegetables, fruits promotes enhanced oxygenation and thereby aids in maintaining the homeostatic regulation. Also the intended results from intermittent fasting suggests an underlying mechanism of enhanced oxygenation. Also its interesting to know the fact that moderate exercise is an antioxidant. It has been observed that exercise causes oxidative damage when its exhaustive. It has been observed that moderate exercise results in activation of underlying signaling pathways that results in useful adaptations in cells. These underlying signaling has shown to up regulate several potential antioxidant enzymes [45]. There lies a similar concept in the 'Vata Raktha Chikitsa', that exhaustive physical activities can have adverse effects in health. This is also suggestive of results obtained in aerobic exercises and yoga. It has been observed that tailored 'Tai -chi' program improved postural instability in patients with Parkinson's disorder [46].

\section{Conclusion}

Oxygen is the mediator of homeostatic regulation. Since the rasayana concept emphasizes tissue regeneration and cell renewal, it can be concluded that the synergistic approaches as used in Ayurvedic treatment methods in 'Vata Vyadi' or 'Vata Rakha Chikitsa' is intended in reestablishing homeostatic regulation by enhancing oxygenation. This can lead to clearance pathways for accumulated dysfunctions, and promotion of non-amyloidogenesis. Enhancing the oxygenation capacity by modifying the oxygen transport and delivery, enhancing factors involving oxygen metabolism can re-establish homeostatic regulation which can further aid in cure and prevention. And hence treatment using nanomedicines capable of enhancing oxygenation can produce outstanding results in progressive neurodegenerative conditions. In the Ayurvedic branch of medicine known as 'Rasa shasthra', the 'Bhasma' which are nanomedicines prepared using traditional methods have been used for centuries. Some of them demonstrates a therapeutic potential resulting in profound hematonergic, immuno modulatory, hemopoietic activity. Moreover the decline in the efficiency of homeostatic regulation due to accumulated dysfunctions can persist and become progressive if the homeostatic regulation is not reestablished. The effects are accumulative over years unless regeneration occurs by re-establishing the disrupted homeostatic regulation. Aging is principally said to be caused by the cumulative damage to tissues by oxygen free radicals and endogenous glycation, which occurs in the body, which is associated with increased oxidative damage. There are underlying mechanisms in human body to counteract oxidative stress by producing antioxidants, which are naturally produced in situ. Through enhancement of oxygenation, progressive loss of dopaminergic neurons in substantia nigra and development of intraneuronal accumulations of aggregated proteins, neuro-fibrillary tangles can be hindered, synaptogenesis and neuroregeneration can be induced which can result in regrowth and repair of nervous tissues and brain cells.

When it comes to neurogenesis, the generation of new neurons occurring in adult mammals as classically described in two defined regions of the brain: the subventricular zone of the lateral ventricles and the subgranular zone of the dentate gyrus, where neural stem cells give rise to new neurons and glia, which functionally integrate into the existing circuits under physiological conditions. This mechanism of neurogenesis also indicates a highly dynamic behavior, which is contributed by promotion of several underlying mechanisms. Experimental studies indicate the presence of neurogenic potential in other brain regions also. Neural progenitor and stem cells have been isolated from various areas of the adult brain, including nonneurogenic areas, such as the spinal cord in species including humans $[47,48]$. This methodology of treating through enhancing oxygenation by modifying the quality, concentration, metabolism, storage and oxygen binding capacity of hemoglobin is also effective in demyelinative conditions. This includes enhancement of factors associated with oxygen metabolism such as oxidases, peroxidases, catalases, hydroxylases, and electron transfer-cytochromes. Upregulation of genetic expression is dependent of oxygen. Genetic predispositions can be corrected by enhancing oxygenation since oxygen is the real time mediator of homeostatic regulation.

When it comes to 'Vata Vyadi', the rasayana concept as explained in classical ayurvedic literature is intended in promoting neurogenesis by reestablishing homeostatic regulation by enhancing oxygenation $[1,10,38-43,49,50]$. This methodology of modulation of neurogenesis by enhancing oxygenation has been followed in ayurvedic medicinal system since thousands of years. And hence it can be concluded that the ayurvedic medicinal approaches towards neurodegenerative conditions and nervous system disorders are mainly focused on enhancing oxygenation. Novel synergistic therapeutic approaches for enhancing oxygenation would thereby aid in reversing neurodegeneration. This novel approach holds the key to the future of treating neurodegenerative conditions, furthermore the future of life sciences. 


\section{References}

1. Text: Ashtangahridayasamhitha, Text: Rasa Tarangini, Ayurveda - Indian System of Medicine.

2. Travis JA, Craddock, Jack A, Tuszynski, Stuart Hameroff, et al. (2012) Cytoskeletal Signaling: Is Memory Encoded in Microtubule Lattices by CaMKII Phosphorylation. Plos pp-2421.

3. Venkatasubramanian G (2015) Understanding schizophrenia as a disorder of consciousness: biological correlates and translational implications from quantum theory perspectives. Clin Psychopharmacol Neurosci 13: 36-47.

4. Hameroff S (2012) How quantum brain biology can rescue conscious free will. Front Integr Neurosci 6: 93

5. Matell MS, Meck WH (2004) Cortico-striatal circuits and interval timing: coincidence detection of oscillatory processes. Brain Res Cogn Brain Res 21: 139-170.

6. Wickstead B, Gull K (2011) The evolution of the cytoskeleton. J Cell Biol 194: 513-525.

7. Fletcher DA, Mullins RD (2010) Cell mechanics and the cytoskeleton Nature 463: 485-492.

8. Gunning PW, Ghoshdastider U, Whitaker S, Popp D, Robinson RC (2015) The evolution of compositionally and functionally distinct actin filaments. J Cell Sci 128: 2009-2019.

9. Jibin J (2015) A Transcending Insight into Disease Etiology and Life Sciences Enhancement of Oxygenation for Reestablishing Homeostasis Governing Life. J Health Edu Res Dev 3: 1-9.

10. Pal D1, Sahu CK1, Haldar A1 (2014) Bhasma : The ancient Indian nanomedicine. J Adv Pharm Technol Res 5: 4-12.

11. Ferrer I, Gómez A, Carmona M, Huesa G, Porta S, et al. (2011) Neuronal hemoglobin is reduced in Alzheimer's disease, argyrophilic grain disease, Parkinson's disease, and dementia with Lewy bodies. J Alzheimers Dis 23: 537-550.

12. Dickson D (2003) Neurodegeneration. The Molecular Pathology of Dementia and Movement Disorders. ISN Neuropath Press, Basel.

13. Uversky VN, Fink A (2007) Protein Misfolding, Aggregation, and Conformational Diseases, Springer, New York.

14. Love S, Louis DN, Ellison DW (2008) Greenfield's Neuropathology, Hodder Arnold, London.

15. Ferrer I, Santpere G, van Leeuwen FW (2008) Argyrophilic grain disease Brain 131: 1416-1432.

16. UNIPROT: P37840, Alpha-Synuclein, Gene: SNCA

17. Bowen RL, Smith MA, Harris PL, Kubat Z, Martins RN, et al. (2002) Elevated luteinizing hormone expression colocalizes with neurons vulnerable to Alzheimer's disease pathology. J Neurosci Res 70: 514-518.

18. Casadesus G, Webber KM, Atwood CS, Pappolla MA, Perry G, et al (2006) Luteinizing hormone modulates cognition and amyloid-beta deposition in Alzheimer APP transgenic mice. Biochim Biophys Acta 1762: 447-452.

19. Bowen RL, Isley JP, Atkinson RL (2000) An association of elevated serum gonadotropin concentrations and Alzheimer disease? J Neuroendocrinol 12: 351-354.

20. Casadesus G, Atwood CS, Zhu X, Hartzler AW, Webber KM, et al. (2005) Evidence for the role of gonadotropin hormones in the development of Alzheimer disease. Cell Mol Life Sci 62: 293-298.

21. Short RA, Bowen RL, O'Brien PC, Graff-Radford NR (2001) Elevated Gonadotropin Levels in Patients with Alzheimer Disease. Mayo Clin Proc 76:906-909.

22. Duman RS (2002) Pathophysiology of depression: the concept of synaptic plasticity. Eup Psy 17: 306-310.

23. Marsden WN (2013) Synaptic plasticity in depression: molecular, cellular and functional correlates. Prog Neuropsychopharmacol Biol Psychiatry 43: 168-184.

24. Husseini Manji K, Orge Quiroz A, J Ennifer Payne L, Jaskaran singh , Barbara Lopes P, et al. (2003) The underlying neurobiology of bipolar disorder. Wpa 2:136-46.
25. Mesulam MM (1999) Neuroplasticity failure in Alzheimer's disease: bridging the gap between plaques and tangles. Neuron 24: 521-529.

26. Davis Parker W, Janice Parks BA, Christopher M, Filley M, KleinschmidtDeMasters BK (1994) Electron transport chain defects in Alzheimer's disease. Cross mark 44: 1090.

27. Pandav S, Vijay Chandra, Hiroko H Dodge (2004) Hemoglobin Levels and Alzheimer Disease: An Epidemiologic Study in India. Ajgp 12: 523 526.

28. John Shoffner M, Ray Watts L, Jorge Juncos L, Antonio Torroni, Douglas Wallace C (1991) Mitochondrial oxidative phosphorylation defects in Parkinson's disease. Ana 30: 332-339.

29. Davis Parker W, Russell Swerdlow H (1998) Mitochondrial Dysfunction in Idiopathic Parkinson Disease. AJHG 62: 758-762.

30. Itoh K, Weis S, Mehraein P, Müller-Höcker J (1997) Defects of cytochrome c oxidase in the substantia nigra of Parkinson's disease: and immunohistochemical and morphometric study. Mov Disord 12: 9-16.

31. Savica R, Grossardt BR, Carlin JM, Icen M, Bower JH, et al. (2009) Anemia or low hemoglobin levels preceding Parkinson disease. Cross Mark 73: 1381-1387.

32. Khristy Thompson J, Shai Shoham, James Connor R (2001) Iron and neurodegenerative disorders. Brain Research Bulletin 55:155-164.

33. Ya Kea, Dr Zhong Ming Qiana (2003) Iron misregulation in the brain: a primary cause of neurodegenerative disorders. Ln 4: 246-253.

34. Megan Whitnall, Des R. Richardson (2006) Iron: A New Target for Pharmacological Intervention in Neurodegenerative Diseases. Ped Nut 13: $186-197$.

35. Vural H, Demirin H, Kara Y, Eren I, Delibas N (2010) Alterations of plasma magnesium, copper, zinc, iron and selenium concentrations and some related erythrocyte antioxidant enzyme activities in patients with Alzheimer's disease. J Trace Elem Med Biol 24: 169-173.

36. Medina DX, Caccamo A, Oddo S (2011) Methylene blue reduces $\mathrm{aI}^{2}$ levels and rescues early cognitive deficit by increasing proteasome activity. Brain Pathol 21: 140-149.

37. Atamna H, Kumar R (2010) Protective role of methylene blue in Alzheimer's disease via mitochondria and cytochrome $c$ oxidase. J Alzheimers Dis 20 Suppl 2: S439-452.

38. Gautam A, Wadhwa R, Thakur MK (2013) Involvement of hippocampal Arc in amnesia and its recovery by alcoholic extract of Ashwagandha leaves. Neurobiol Learn Mem 106: 177-184.

39. Gautam A, Kaul SC, Thakur MK (2015) Alcoholic Extract of Ashwagandha Leaves Protects Against Amnesia by Regulation of Arc Function. Mol Neurobiol .

40. Winters $M$ (2006) Ancient medicine, modern use: Withania somnifera and its potential role in integrative oncology. Altern Med Rev 11: 269-277.

41. Mishra LC, Singh BB, Dagenais S (2000) Scientific basis for the therapeutic use of Withania somnifera (ashwagandha): a review. Altern Med Rev 5: 334-346.

42. Teresa Koby (2011) Effects of Withania somnifera (Ashwagandha) and Terminalia arjuna (Arjuna)on physical performance and cardiorespiratory endurance in healthy young adults.HRF.

43. Raghavan A, Shah ZA1 (2015) Withania somnifera: a pre-clinical study on neuroregenerative therapy for stroke. Neural Regen Res 10: 183-185.

44. Gruber R, Axmann S, Schoenberg MH (2005) The influence of oxygenated water on the immune status, liver enzymes, and the generation of oxygen radicals: a prospective, randomised, blinded clinical study. Clin Nutr 24: 407-414.

45. Gomez-Cabrera MC, Domenech E, Viña J (2008) Moderate exercise is an antioxidant: upregulation of antioxidant genes by training. Free Radic Biol Med 44: 126-131.

46. Li F, Harmer P, Fitzgerald K, Eckstrom E, Stock R, et al. (2012) Tai chi and postural stability in patients with Parkinson's disease. N Engl J Med 366: 511-519. 
Citation: Jibin Joy (2015) The Ayurvedic Perspective on the Etiopathogenesis of Neurodegeneration. J Health Edu Res Dev 3: 137. doi: $10.4172 / 2380-5439.1000137$

Page 5 of 5

47. Oyarce K, Bongarzone ER, Nualart F (2014) Unconventional Neurogenic Niches and Neurogenesis Modulation by Vitamins. J Stem Cell Res Ther 4: 184.

48. Taupin P, Gage FH (2002) Adult neurogenesis and neural stem cells of the central nervous system in mammals. J Neurosci Res 69: 745-749.
49. Joshi KS, Bhonde R2 (2014) Insights from Ayurveda for translational stem cell research. J Ayurveda Integr Med 5: 4-10.

50. Rammohan Rao V, Olivier Descamps, Varghese John, Dale Bredesen E (2012) Ayurvedic medicinal plants for Alzheimer's disease: a review. BMC $4: 22$. 